\title{
Social-Skill Interventions for Culturally and Linguistically Diverse Students with Disabilities: A Comprehensive Review
}

\author{
Sunyoung Kim \\ University of Alabama-Tuscaloosa \\ Min-Chi Yan \\ Northeastern State University-Broken Arrow \\ Saili S. Kulkarni \\ California State University-Dominguez Hills
}

\begin{abstract}
Teachers and researchers have considered social-skill interventions to be an essential component in the development and progress of students with disabilities. However, there is still relatively limited research on these interventions for individuals from culturally and linguistically diverse (CLD) backgrounds. This literature review was conducted to examine the effectiveness of social-skill interventions for CLD students with disabilities in school settings. Electronic database searches and a manual search were completed to identify studies published between 2000 and 2017 (February). Seven studies ( $\mathrm{n}=18$ participants) were identified for inclusion in this review, and five types of social interventions were identified. Most participants were male, aged between 8 and 13 years old, were considered at risk for having developmental delay or had developmental delay, and were identified as African Americans. The majority of studies we reviewed utilized single-subject research designs and focused on social interactions as the goal for their individual interventions. Peer-mediated interventions and social story intervention were the most frequently used interventions. Findings suggest that, when exposed to the social-skill interventions, CLD children with disabilities improved their social behaviours and skills. Some children with disabilities maintained and generalized these behaviours across settings or playmates.
\end{abstract}


Acquiring social skills and competence is unarguably important in children's development (Gresham, Sugai, \& Horner, 2001). Social competence and ability can assist in establishing interpersonal relationships and bonds (Gresham et al., 2001). Students with disabilities, however, are often reported as lacking such social behaviours regardless of the severity and diagnosis of disabilities and their level of intellectual functioning (Miller, Lane, \& Wehby, 2005). These difficulties in social skills also interfere with their academic learning and limit their occupational progress (Cullinan, 2002; Howlin \& Goode, 1998).

In school settings, students with disabilities often face with specific social-skill challenges, including initiating and maintaining conversations and playing with peers (Banda, Hart, \& LiuGitz, 2010; Terpstra, Higgins, \& Pierce, 2002). Although school settings offer numerous opportunities for social interactions (Harper, Symon, \& Frea, 2008), without appropriate interventions, students with disabilities may be more likely to become isolated than their typically developing peers. For the example of students with autism spectrum disorders (ASD), Chamberlain, Kasari, and Rotherham-Fuller (2007) reported that when integrated with typically developing peers in inclusive classrooms, students with ASD are at increased risk for peer rejection and social isolation because of their social difficulties. Accordingly, along with focusing on other developmental domains, improving social skills for students with disabilities is a critical goal for teachers, and effective social-skill interventions should be considered a priority in the design of educational programs for students with disabilities (Bellini, Peters, Benner, \& Hopf, 2007).

The number of culturally and linguistically diverse (CLD) students with disabilities has steadily increased in the United States; CLD students comprise the majority of the population of students with disabilities in the nation (U.S. Department of Education, 2009). However, in school, educators are often facing an escalating challenge resulting from understanding and addressing cultural and linguistic differences:

\section{Such significant concerns have resulted in a lack of understanding of the needs of students who are English language learners, ineffective behavioral interventions to assist struggling students from diverse backgrounds, and the inability to accurately differentiate between cultural differences and genuine disability indicators. (Moreno, Wong-Lo, Short, \& Bullock, 2014, p. 344)}

As Cartledge and Loe (2001) stressed, individuals demonstrate culturally relevant behaviours because "all behavior is culturally based" (p. 33). However, social behaviours of CLD students are often seen through an incongruent cultural lens in schools and require an in-depth understanding of the interactions between cultural identities (Cartledge \& Loe, 2001). Particularly, these culturally relevant social skills may have an impact on their behavioural and/or academic performance when they interact with their teachers and peers in school settings. Thus it is imperative for educators to provide culturally sensitive social-skill interventions for their CLD students with disabilities who usually experience social challenges as a result of the disability and/or cultural difference.

An extensive body of intervention literature has evaluated instructional strategies and support for addressing the social skills (e.g., social interactions and social engagement) in school settings. However, little is known about what kinds of culturally responsive social-skill interventions are 
available and accessible for CLD school-aged children with disabilities. Especially given the disproportionate representation of minority students in special education and the inequity of intervention services of CLD students with disabilities across racial groups (Skiba et al., 2008; Waitoller, Artiles, \& Cheney, 2010), it is vital for researchers and educators to investigate the accessibility and feasibility of school-based social-skill interventions that are culturally responsive to meet the behavioural needs of the CLD students with disabilities. Culturally responsive teaching is understood as a collection of best teaching practices that enhance the academic success of CLD students in classroom settings (Gay, 2002; Ladson-Billings, 1994).

The purpose of the present review was to synthesize empirically based studies with a focus on assessing the cultural responsiveness of social-skill interventions for CLD students with disabilities or who are at risk for developing disabilities. Our focus was on how CLD students with disabilities have been socially supported in school settings. Based on our findings, we discuss guidelines and potential directions for future researchers and practitioners.

\section{Method}

\section{Inclusion and Exclusion Criteria}

Studies included in this literature review were based on the following seven criteria. Each study (a) included participants ages 3-18 years old with a disability or at risk of having a disability; (b) included participants from CLD backgrounds; (c) was conducted utilizing experimental designs (e.g., single-case research design and randomized controlled trials) or descriptive designs that presented clear behaviour outcomes associated with the interventions; (d) implemented an intervention in an effort to teach student participants social skills or social behaviours; (e) took place within the context of a school; (f) was published in a peer-reviewed journal between 2000 and 2017 (February); and (g) was published in English.

\section{Search Procedures}

Electronic searches were completed using electronic databases such as ERIC, PsychINFO, Wilson, Academic Search Premier, and MEDLINE. Searches were carried out using the following five levels of search terms: Level 1: culturally responsive OR culturally relevant $O R$ culturally sensitive OR cultural consideration OR cultural awareness; Level 2: social skills OR socialization OR social behavior OR social competence OR social problem solving OR social interaction; Level 3: interventions OR programs OR curriculum OR treatment; Level 4: students OR learners OR child OR children OR youth OR adolescents OR teenagers; and Level 5: disabilit*. Additionally, we completed a manual search of five journals, including Behavioral Disorders, Exceptionality, International Journal of Special Education, Journal of Positive Behavior Interventions, and Journal of Autism and Developmental Disorders (2011 to 2017 [February]) that had published the studies that were already included for review for possible additions to the review. We also examined the reference sections of each selected study, seeking possible additions to the review. 
Table 1

A Summary of Demographic Information and Results

\begin{tabular}{|c|c|c|c|c|c|c|c|c|c|}
\hline \multirow[b]{2}{*}{ Citations } & \multicolumn{4}{|c|}{ Participant Characteristics } & \multirow{2}{*}{ Intervention } & \multirow[b]{2}{*}{ Goal } & \multirow[b]{2}{*}{ Results } & \multirow{2}{*}{$\begin{array}{l}\text { Val. I } \\
\text { Fid. }\end{array}$} & \multirow{2}{*}{$\begin{array}{l}\text { Main. I } \\
\text { Gen. }\end{array}$} \\
\hline & $\begin{array}{c}\text { Agel } \\
\text { Gender }\end{array}$ & Diagnosis & $\begin{array}{c}\text { Racel } \\
\text { Ethnicity }\end{array}$ & SES & & & & & \\
\hline $\begin{array}{l}\text { Bardon, Dona, \& Symons } \\
\text { (2008) }\end{array}$ & $\begin{array}{c}8-9 \\
2 \text { girls, } 1 \text { boy }\end{array}$ & $\begin{array}{l}\text { At risk/ } \\
\text { ADHD }\end{array}$ & AA & $\mathrm{N} / \mathrm{A}$ & PATHS & Interactions & $\mathrm{P}$ & $\mathrm{Y} / \mathrm{N}$ & $N / Y$ \\
\hline $\begin{array}{l}\text { Blake, Wang, Cartledge, \& } \\
\text { Gardner (2000) }\end{array}$ & $\begin{array}{l}12-13 \\
2 \text { boys }\end{array}$ & SED & AA & Low & Peer-mediation & Interactions & $\mathrm{P}$ & $\mathrm{Y} / \mathrm{Y}$ & $\mathrm{Y} / \mathrm{N}$ \\
\hline $\begin{array}{l}\text { Delano \& Snell } \\
(2006)\end{array}$ & $\begin{array}{c}6 \\
1 \text { boy }\end{array}$ & ASD & AA & $\mathrm{N} / \mathrm{A}$ & Social Story & $\begin{array}{c}\text { Social } \\
\text { engagement }\end{array}$ & $\mathrm{P}$ & $\mathrm{N} / \mathrm{N}$ & $\mathrm{Y} / \mathrm{Y}$ \\
\hline $\begin{array}{l}\text { Harper, Symon, \& Frea } \\
(2008)\end{array}$ & $\begin{array}{c}8 \\
1 \text { boy }\end{array}$ & ASD & $\begin{array}{c}\text { Asian } \\
\text { (Vietnamese) }\end{array}$ & $\mathrm{N} / \mathrm{A}$ & $\begin{array}{l}\text { Pivotal response } \\
\text { training }\end{array}$ & Interactions & $\mathrm{P}$ & $\mathrm{Y} / \mathrm{Y}$ & $N / Y$ \\
\hline $\begin{array}{l}\text { Hsu, Hammond, \& Ingalls } \\
(2012)\end{array}$ & $\begin{array}{c}3-6 \\
3 \text { boys }\end{array}$ & $\begin{array}{l}\text { ASD/ } \\
\text { DD }\end{array}$ & Hispanic & $\mathrm{N} / \mathrm{A}$ & Social story & Interactions & $P$ & $N / Y$ & $\mathrm{~N} / \mathrm{N}$ \\
\hline $\begin{array}{l}\text { Lee, Odom, \& Loftin } \\
\text { (2007) }\end{array}$ & $\begin{array}{c}9 \\
1 \text { boy }\end{array}$ & $\begin{array}{l}\text { ASD/ } \\
\text { ID }\end{array}$ & $\mathrm{AA}$ & $N / A$ & $\begin{array}{l}\text { Peer-mediated } \\
\text { intervention }\end{array}$ & $\begin{array}{c}\text { Social } \\
\text { engagement }\end{array}$ & $\mathrm{P}$ & $Y / Y$ & $N / Y$ \\
\hline $\begin{array}{l}\text { Lo, Mustian, Brophy, \& } \\
\text { White (2011) }\end{array}$ & $\begin{array}{l}8-12 \\
7 \text { boys }\end{array}$ & $\begin{array}{l}\mathrm{LD} / \mathrm{ID} / \\
\text { at risk }\end{array}$ & AA & $\mathrm{N} / \mathrm{A}$ & $\begin{array}{l}\text { Peer-mediated } \\
\text { Intervention }\end{array}$ & Interactions & $\mathrm{P}$ & $\mathrm{Y} / \mathrm{Y}$ & $\mathrm{Y} / \mathrm{N}$ \\
\hline
\end{tabular}

Notes. $A A=$ African American, $A D H D=$ attention-deficit/hyperactivity disorder, $A S D=$ autism spectrum disorders, $D D=$ developmental delay Fid. = Fidelity,$\quad$ Gen. = Generalization,$\quad I D=$ intellectual disability, $\quad L D=$ learning disabilities, Main. = Maintenance, $\quad N=$ no, $\quad P=$ positive, PATHS = Promoting Alternative Thinking Strategies, SED = serious emotional disturbances, Val. = Validity, and $\mathrm{Y}=$ yes . 
In the initial database search, two empirical articles were identified from a total of 81 articles that matched the search criteria. To ensure reliability of researching outcomes, we separately reviewed the 81 articles and reached $100 \%$ agreement. One of us (Kim) reviewed all of the 81 articles, and Yan and Kulkarni reviewed 41 and 40 articles, respectively, for the reliability check. The reliability was calculated by having the total number of agreements divided by total number of agreements plus disagreements. An additional five articles were found through the manual search. The total number of articles that were examined for this literature review included seven empirical articles, including 18 participants.

\section{Data Analysis}

Table 1 shows a summary of each study included for this review. Two of us (Kim and Yan) independently reviewed the seven studies and manually coded the information using the variables indicated in the table. The two sets of data met $100 \%$ agreement across the six variables (i.e., the total number of agreements divided by total number of agreements plus disagreements).

\section{Results}

\section{Participant Characteristics}

Each of the seven studies included in this review reported varying participant characteristics, in areas of age, gender, disability diagnosis, race, and/or socioeconomic status. With respect to gender, most of the 18 participants across all seven studies identified as male $(n=16 ; 89 \%)$. Participants ranged from 3 to 13 years, but the majority of participants $(n=14 ; 78 \%)$ were between 8 and 13 years old.

The types of disabilities presented in the studies varied. Nine participants were considered at risk for having developmental delay or had developmental delay $(50 \%)$, and four students had ASD (i.e., autism, pervasive developmental disorder, or Asperger's syndrome; 22\%). Two students had emotional and behavioural disorders (11\%), one student had intellectual disabilities, and another student had a learning disability. The remaining student had attention-deficit/hyperactivity disorder. In terms of racial/ethnic backgrounds, the majority of the participants were African American $(n=14 ; 78 \%), 17 \%$ were Hispanic $(n=3)$ and $5 \%$ were Vietnamese $(n=1)$. Only one out of the seven studies reported socioeconomic status of their participants (Blake, Wang, Cartledge, \& Gardner, 2000), which was low.

\section{Research Designs}

Six of the seven studies utilized single-subject research designs and the remaining one study utilized a descriptive study design with visible results of behaviour development.

\section{Targeted Goals}

Five studies focused on social interactions as the goal for their individual interventions, and the remaining two studies focused on social engagement (Delano \& Snell, 2006; Lee, Odom, \& Loftin, 2007). In Delano and Snell's (2006) study, the duration of social engagement and the frequency of four social skills were examined, including 
seeking attention, initiating comments, initiating requests, and making contingent responses. Lee et al. (2007) examined the relationship between engagement in social interactions with peers and stereotypic behaviours (e.g., hand flopping, head rolling).

\section{Intervention Strategies}

Peer-mediated intervention. Three studies implemented peer-mediated interventions to increase positive social-skill interactions for students with disabilities (Blake et al., 2000; Lee et al., 2007; Lo, Mustian, Brophy, \& White, 2011). Lee et al.'s (2007) study also employed a peer-mediated approach to increase social interactions for an elementary African-American student with ASD. This intervention specifically monitored the percentage of time the student with ASD interacted socially with his peers. Typically developing peers were trained to engage in social interaction with the student with ASD. The peer training provided a foundation that helped increase positive social interactions between the student with ASD and the peers. The results indicated that having peers engage in social interaction promoted positive outcomes in decreasing stereotypic behaviours for the participant with ASD.

In Lo et al.'s (2011) study, the authors implemented culturally responsive Social Skill Instruction (SSI), a peer-mediated program for elementary African-American students with or at risk for mild disabilities (e.g., learning disabilities and intellectual disabilities). Peer tutors from the participants' cultural group were trained individually to conduct the scripted lessons using a training script. The findings showed that all participants increased their skills and knowledge of classroom-related and aggressionresolution social skills and that the majority of participants decreased negative classroom behaviours during the intervention.

Blake et al. (2000) implemented peer-mediated interventions for middle school African-American students with serious emotional disturbances. Students with serious emotional disturbances were selected as peer tutors, trained on how to use the materials, and given scripts with the necessary steps. Then the peer tutors implemented the socialskill intervention for other students with serious emotional disturbances, using folktales for introducing the appropriate social behaviours, modeling new social-skill behaviours, and doing extension activities. Their findings indicated the desired effects of the peerbased social-skill instruction, with some evidence of behaviour maintenance and transfer.

Social story intervention. Two studies integrated social stories into their interventions to facilitate students' social-skill learning (Delano \& Snell, 2006; Hsu, Hammond, \& Ingalls, 2012). Delano and Snell (2006) used a social storytelling approach in their intervention for the improvement of social engagement for elementary AfricanAmerican students with autism. Those target children were paired with same-age peers without disabilities. After the peer and target child listened to the social stories, they were told to play for 10 minutes. Findings showed that the use of social stories was effective in promoting the duration of social engagement and the frequency of specific social skills including initiating comments and requests, making responses, and seeking attention (Delano \& Snell, 2006).

Similarly, Hsu et al. (2012) employed a culturally based social story technique in their intervention to promote social interactions for elementary Hispanic students with 
developmental delay and autism. The effects of using the basic social stories without cultural elements and using multicultural social stories were compared. The multiculturally relevant components in the home-language version of stories included culturally relevant illustrations (e.g., characters) and names. Findings indicated that use of social story interventions generally provided a positive impact on behaviour changes in all participants. However, the level of effectiveness of using stories with culturally relevant elements varied across those participants: Of the three children in the study, one child showed better outcomes with culturally relevant social stories than with the basic social stories written in English, one showed a lower level of outcomes with culturally relevant stories, and one did not demonstrate any difference in outcomes between the two different types of stories.

Pivotal response training. One study used peer-mediated pivotal response training (PRT) to increase social interactions for an elementary Vietnamese student with autism during recess activities (Harper et al., 2008). Harper et al. (2008) recruited typically developing students from the same classroom of the target student with autism and provided those peers with training following PRT manuals (Pierce \& Schreibman, 1997). The peers as mediators were taught to say the name of a student and give a prompt to "look and listen" while making eye contact with the student, to offer play options, to praise, and to model play for the student with autism. Then the peers met the target student with autism and used the PRT strategies when they were told to play during recess. After the peer-training session, the social interaction between the target student with autism and the peers was observed. Harper et al.'s findings showed that the target participant improved his social peer interaction during recess following a peerimplemented PRT intervention and demonstrated positive generalization of the acquired skills to a new setting.

PATHS curriculum. One study utilized the Promoting Alternative Thinking Strategies (PATHS) curriculum, a research-based violence-prevention program, to increase social interaction and co-operative play of elementary African-American students at risk for being identified as having emotional and behavioural disorders and attention-deficit/hyperactivity disorder (Bardon, Dona, \& Symons, 2008). PATHS curriculum, a K-6 class-wide program, is designed to (a) promote social and emotional competence in children, (b) reduce aggression and acting-out behaviours, and (c) enhance the educational process for all students. First, the researchers trained general educators to use PATHS. Those teachers implemented the PATHS curriculum by delivering explicit instruction on PATHS lessons over a 10-week period and observed the behaviour of their student participants. The findings indicated that all participants demonstrated positive social interactions with others and increased co-operative play after using PATHS.

\section{Intervention Outcomes}

In terms of improving social skills, outcomes across studies were related to positive gains in several social skills including social engagement in all participants.

Maintenance and generalization. Three studies included the collection of maintenance data and reported treatment effects from one to four weeks following the intervention (Blake et al., 2000; Delano \& Snell, 2006; Lo et al., 2011). These studies 
reported positive maintenance effects. In addition, four studies assessed generalization across one condition (i.e., novel settings or peers) and reported positive generalization (Bardon et al., 2008; Delano \& Snell, 2006; Harper et al., 2008; Lee et al., 2007). The generalization procedure was identical to baseline in most studies.

Social validity. Five of the seven studies reported assessing the social validity of their interventions, which measured the degree of social acceptability of goal, procedure, and outcomes of the interventions (Bardon et al., 2008; Blake et al., 2000; Harper et al., 2008; Lee et al., 2007; Lo et al., 2011). Overall, all of the five studies that integrated the component of social validity showed positive results. Social validity was assessed by evaluating the results of questionnaires, journals, or interviews completed by a variety of stakeholders (i.e., teachers, researchers, and students) for these social-skill intervention studies. Of these five studies, three used questionnaires or a rating scale to report social validation (Blake et al., 2000; Lee et al., 2007; Lo et al., 2011); one study measured social validity using teacher journals (Bardon et al., 2008); and one study used anecdotal reports to assess the social validity (Harper et al., 2008).

Treatment fidelity. Five of the seven studies provided a measure of treatment fidelity for their social-skill interventions, in order to assess the degree to which all intervention sessions were executed according to protocol (Blake et al., 2000; Harper et al., 2008; Hsu et al., 2012; Lee et al., 2007; Lo et al., 2011). In the five studies, all observers and the interventionists completed a procedural fidelity checklist outlining the procedure for each session. Those checklists indicated whether interventionists or peer-trainers followed the intervention protocols and systematically implemented the intervention procedures or steps, and/or whether the target children had difficulties attending to the interventions. In most studies, the observers evaluated the fidelity by reviewing $30 \%$ of all sessions for each participant. All studies reported high levels of fidelity.

\section{Discussion}

Given the increased number of CLD students in the United States, considering cultural and social responsiveness in intervention studies has been highlighted as a necessary task for intervention researchers (Bal \& Trainor, 2016). In the current literature review, we investigated the effectiveness of social-skill interventions for CLD students with disabilities or at risk for disability diagnosis in school settings. The outcomes of the seven studies reviewed suggest that each social-skill intervention was effective in addressing the development of social skills for CLD students with disabilities or at risk for disabilities. Indeed, for all of the participants, the interventions built targeted social behaviours and facilitated the maintenance of the acquired behaviours over time. A number of discussion points were identified: natural agents of interventions, cultural responsiveness, intervention strategies, and goals of social-skill interventions.

\section{Natural Agents of Interventions}

As social behaviours are defined as learned behaviours and skills that are socially accepted by cultural groups (Cartledge \& Loe, 2001), interventionists as social-skill guidance or play/interaction partners may play an important role in students' initial learning. We found that of the seven studies, three studies used adults as delivery agents, 
including a general education teacher, a special education teacher, an early childhood interventionist, and a researcher.

In schools, teachers can be effective natural change agents in intervention studies (Glenwick \& Barocas, 1979). Compared to research staff, teachers are more knowledgeable about their students and can continuously implement interventions in schools. Additionally, the nature of social interactions between teachers and CLD students is personal and value laden (Cartledge \& Milburn, 1996; Franklin, 1992). Although the research articles analyzed for this review did not provide details about how the teachers addressed the needs of those students with CLD and modified the intervention accordingly, the most important lesson for successful implementation of social-skill intervention may be whether these teachers are culturally sensitive to their students with CLD backgrounds.

For students with disabilities, peers might be the most preferable play partners over adults because peers are less likely to interrupt the nature of social exchange of their interactive behaviour (e.g., play; Strain, 2001). Compared to adults, peers also are able to provide a more natural facilitation of play. Of the seven studies included for this review, four studies adopted peers as intervention mediators (i.e., peers with and without disabilities; Blake et al., 2000; Harper et al., 2008; Lee et al., 2007; Lo et al., 2011). Particularly for CLD participants with disabilities, Lo et al.'s (2011) study included peer mediators from the target students' cultural group, in order to better address and promote social competency of African-American students with disabilities. The intervention procedures and curriculum also related to the students' real life experiences (in and out of school), personal stories, and materials (e.g., diverse racial as well as African-American characteristics). In other words, peers' and target children's culture has been embedded into the interventions for the students with CLD backgrounds. Peer mediations as an established intervention seem promising for promoting social competence of students with special needs as well as for increasing the notion of inclusion for CLD students with disabilities.

\section{Cultural Responsiveness}

As noted in the introduction, this study used a broad definition of cultural responsiveness from Gay (2002) as situating the academic experiences of CLD students within their cultural experiences, perspectives, and characteristics. To understand how the articles reviewed addressed cultural responsiveness, we used broad terms in searches such as culturally competent and culturally relevant, and reviewed studies that included CLD populations. As a result, we reviewed articles that included participants from different cultural and racial groups such as African-American students (78\%), Hispanic students (17\%), and an Asian-American student (5\%). Blake et al. (2000) and Lo et al. (2011), in particular, included African-American students. Hsu et al. (2012) specifically mentioned the use of culturally responsive strategies (i.e., using relevant social stories and home language) to include Hispanic students with disabilities. Studies documented other important student characteristics in order to understand students' backgrounds and embed them into the practices. For instance, Lo et al. (2011) also provided characteristics such as the type of disability, family history, and behavioural referrals. Most studies, however, provided limited information about the participants' characteristics or intersections of cultural factors such as race, ability, and socioeconomic status. 
Careful consideration of the differences and similarities in social skills arising from the varied cultural interaction styles in diverse classrooms has been suggested as a first step in designing culturally responsive interventions (Artiles, Kozleski, Trent, Osher, \& Ortiz, 2010). Therefore, social-skill interventions for CLD students need to include diverse perspectives in the contexts of the community, cultural, school, family, and systemic levels from the state to the city to the neighborhood (Artiles et al., 2010). An analysis of the learned behaviours of participants throughout their complex social interactions (i.e., family, peer, community, and teacher) were not considered a part of each study. Furthermore, there was not sufficient information on any language barriers and no indication of whether participants were placed in English Language Learner programs. Additionally, there was limited discussion regarding school institutional history and the effects on the participants' social-skill behaviour. These cultural tools (e.g., interactional styles, cultural history, and language) that mediate social behaviours may be an important factor to consider in culturally responsive interventions (Engeström, 2001). Findings of the studies showed positive results, but little has been recorded regarding the complexity of language, culture, and race that is embedded in social-skill training. These areas are critical to future research in the area of social-skill interventions for students with disabilities from CLD backgrounds.

\section{Intervention Strategies}

Our literature review found several effective intervention strategies that promoted social skills for CLD students with diverse disabilities. The most frequently used intervention strategies were peer-mediated interventions. Several other studies have shown benefits of using peers as change agents (e.g., peer tutors) in increasing the social interactions of children with disabilities (e.g., Gresham et al., 2001; Lo, Loe, \& Cartledge, 2002; Maag, 2005, 2006; Miller et al., 2005; Prater, Serna, \& Nakamura, 1999; Spence, 2003). It is fortunate to find that the use of peer-mediated interventions is also effective to support CLD students with disabilities. Particularly, it is meaningful that the CLD participants with disabilities experienced successful within- and cross-cultural peer relationships with the interventions and that such interventions yielded socially and statistically valid outcomes.

However, as many researchers have highlighted, the acceptability and generalizability of evidence-based social behavioural interventions should be examined within diverse cultural communities (Artiles et al., 2010). There should be sufficient evidence to support social-skill development of CLD students with the existing evidencebased interventions. Specifically, there should be more examination of how the assorted intervention strategies can fit diverse contextual needs of CLD students with disabilities.

\section{Goals of Social-Skill Interventions}

The majority of studies $(n=5 ; 71.4 \%)$ reviewed focused on the improvement of social interactions for CLD students with disabilities. Students with CLD backgrounds and disabilities might experience multiple layers of complexity while socially interacting with their peers. This might be due to their culturally relevant behaviour (Cartledge \& Loe, 2001) in conjunction with the nature of the disability they have. The attention to social interaction skills in the studies reviewed implies that compared to their mainstream 
peers with or without disabilities, CLD students with disabilities are in increased and unique need of research-based support (i.e., effective social-skill interventions).

\section{Limitations and Implications}

This study was subject to a number of limitations. First, this literature review followed a systematic approach and did not include non-peer-reviewed journal articles, dissertations, and technical reports. Therefore, it is likely that the positive effectiveness of social-skill interventions reported may be inflated as a result of publication bias. Additionally, only seven studies were located that met the criteria for the literature review. The relatively small number of studies included in our review reflects a scarcity of empirical information on the efficacy of social-skill interventions for CLD students with disabilities. This also demonstrates the need for more empirical research in this area for diverse learners with disabilities.

\section{Development of Social Skill and Social Competency}

This review has several implications for educational practices and research. First, CLD students with disabilities or at risk for disabilities positively responded to their individualized social-skill interventions. This indicated that these students had the potential to improve their social skills with appropriate support of social-skill interventions. Such social development can be the foundation of social competency, defined as "the ability to respond appropriately in social contexts, and to meet the cognitive, physical, and linguistic demands of a social context as judged by significant others in that environment" (Hune \& Nelson, 2002, p. 195; Koegel, Koegel, \& Dunlap, 1996). The constant exposures to positive social experiences with interventions may promote the development of social competence that would better support CLD students to experience academic and behavioural success in and out of schools across cultural contexts. Although we found limited details about how these learners with CLD backgrounds met the cognitive, physical, and linguistic demands of a social context, their positive results maybe provide preliminary evidence necessary for future studies to support students using culturally responsive interventions. Future studies might also focus on applying diverse intervention strategies to see their cultural responsiveness within CLD student populations.

\section{Diverse Natural Agents}

The studies in this review recruited diverse implementers in schools (e.g., general education teachers, special education teachers, or research staff). Use of such natural agents successfully generated positive outcomes in the school settings. Future practitioners may consider the involvement of other immediate social contexts such as parents, siblings, and community partners in social-skill interventions within various educational settings. For instance, families may be the most effective and resourceful natural change agents since they are the major caregivers for students and share similar cultural values and linguistic resources. In addition, parents and siblings can generalize the use of social-skill interventions to other settings outside schools such as the home or community setting. Thus practitioners and researchers' efforts to understand family values or reflect them with respect to intervention planning should first be considered 
(Trainor \& Bal, 2014). Additionally, children's social experiences with their peers are important. Providing play opportunities and interactions with peers in the children's immediate ecology (e.g., school and community) may help promote within- and crosscultural social competency.

\section{Social Acceptability and Validity}

Most studies included in this review used single-subject designs, and many of the studies reported social validity with questionnaires and rating scales. According to Kennedy (2005), social validity is the estimation of the effectiveness, appropriateness, importance, and/or satisfaction of various people in relation to a particular intervention. Horner et al. (2005) identified that social validity measurement is a quality indicator for rigorous single-subject research. Wolf (1978) also suggests that since the educational research occurred in applied contexts, knowing how people in applied settings react to an intervention is an important component of understanding the effects of a behavioural intervention. Particularly, researchers of CLD students with disabilities should consider social and cultural differences or diversity when they design an intervention, set a goal, and deliver the intervention. Thus in each study reviewed, even though most participants exhibited changes that aligned with the introduction of the intervention, a measure of social validity involving stakeholders' attribution of these changes in-depth may add strength to the results and provide culturally relevant interpretations of the results.

Finally, further social-skill intervention research needs to include a more complex understanding of cultural differences and the interconnected relationship between culture, educational context, family, teacher, administration, and peers, which shape a child's social interactions (Bal \& Trainor, 2016). Future research may combine the different elements of how students learn social behaviours and factors, which produce ecologically valid interventions that address complex issues. Diverse investigations of social validity may be also required (e.g., in-depth interviews with diverse stakeholders).

\section{References}

Artiles, A. J., Kozleski, E. B., Trent, S. C., Osher, D., \& Ortiz, A. (2010). Justifying and explaining disproportionality, 1968-2008: A critique of underlying views of culture. Exceptional Children, 76(3), 279-299.

Bal, A., \& Trainor, A. A. (2016). Culturally responsive experimental intervention studies: The development of a rubric for paradigm expansion. Review of Educational Research, 86(2), 319-359.

Banda, D. R., Hart, S. L., \& Liu-Gitz, L. (2010). Impact of training peers and children with autism on social skills during center time activities in inclusive classrooms. Research in Autism Spectrum Disorders, 4, 619-625.

*Bardon, L. A., Dona, D. P., \& Symons, F. J. (2008). Extending classwide social skills interventions to at-risk minority students: A preliminary application of randomization tests combined with single-subject methodology. Behavioral Disorders, 3(33), 141-152.

Bellini, S., Peters, J. K., Benner, L., \& Hopf, A. (2007). A meta-analysis of school-based social skills interventions for children with autism spectrum disorders. Remedial and Special Education, $28(3), 153-162$. 
*Blake, C., Wang, W., Cartledge, G., \& Gardner, R. (2000). Middle school students with serious emotional disturbances serve as social skills trainers and reinforcers for peers with SED. Behavioral Disorders, 25(4), 280-298.

Cartledge, G., \& Loe, S. A. (2001). Cultural diversity and social skill instruction. Exceptionality, 9(1), 33-46.

Cartledge, G., \& Milburn, J. F. (1996). Cultural diversity and social skills instruction: Understanding ethnic and gender differences. Champaign, IL: Research Press.

Chamberlain, B., Kasari, C., \& Rotherham-Fuller, E. (2007). Involvement or isolation? The social networks of children with autism in regular classrooms. Journal of Autism and Developmental Disorder, 37, 230-242.

Cullinan, D. (2002). Students with emotional and behavioral disorders: An introduction for teachers and other helping professionals. Upper Saddle, NJ: Prentice Hall.

*Delano, M., \& Snell, M. E. (2006). The effects of social stories on the social engagement of children with autism. Journal of Positive Behavior Interventions, 8(1), 29-42.

Engeström, Y. (2001). Expansive learning at work: Toward an activity theoretical reconceptualization. Journal of Education and Work, 14(1), 133-156.

Franklin, M. E. (1992). Culturally sensitive instructional practices for African-American learners with disabilities. Exceptional Children, 59(2), 115-122.

Gay, G. (2002). Preparing for culturally responsive teaching. Journal of Teacher Education, 53(2), $106-116$.

Glenwick, D. S., \& Barocas, R. (1979). Training impulsive children in verbal self-control by use of natural change agents. The Journal of Special Education, 13(4), 387-398.

Gresham, F. M., Sugai, G., \& Horner, R. H. (2001). Interpreting outcomes of social skills training for students with high-incidence disabilities. Exceptional Children, 67(3), 331-344.

*Harper, C. B., Symon, J. B. G., \& Frea, W. D. (2008). Recess is time-in: Using peers to improve social skills of children with autism. Journal of Autism and Developmental Disorders, 38, $815-826$.

Horner, R. H., Carr, E. G., Halle, J., McGee, G., Odom, S., \& Wolery, M. (2005). The use of singlesubject research to identify evidence-based practice in special education. Exceptional Children, 71(2), 165-179.

Howlin, P., \& Goode, S. (1998). Outcomes in adult life for people with autism, Asperger syndrome. In F. R. Volkmar (Ed.), Autism and pervasive developmental disorders (pp. 209-241). New York, NY: Cambridge University Press.

*Hsu, N., Hammond, H., \& Ingalls, L. (2012). The effectiveness of culturally based social stories to increase appropriate behaviors of children with developmental delays. International Journal of Special Education, 27(1), 104-116.

Hune, J. B., \& Nelson, C. M. (2002). Effects of teaching a problem-solving strategy on preschool children with problem behavior. Behavioral Disorders, 27(3), 185-207.

Kennedy, C. H. (2005). Single-case designs for educational research. Boston, MA: Pearson Education.

Koegel, L. K., Koegel, R. L., \& Dunlap, G. (1996). Positive behavioral support: Including people with difficult behavior in the community. Baltimore, MD: P. H. Brookes.

Ladson-Billings, G. (1994). The dreamkeepers. San Francisco, CA: Jossey-Bass Publishing Co.

*Lee, S., Odom, S. L., \& Loftin, R. (2007). Social engagement with peers and stereotypic behavior of children with autism. Journal of Positive Behavior Interventions, 9(2), 67-79. 
Lo, Y.-Y., Loe, S. A., \& Cartledge, G. (2002). The effects of social skills instruction on the social behaviors of students at risk for emotional or behavioral disorders. Behavioral Disorders, 27, $371-385$.

*Lo, Y., Mustian, A. L., Brophy, A., \& White, R. B. (2011). Peer-mediated social skill instruction for African American males with or at risk for mild disabilities. Exceptionality, 19(3), 191-209.

Maag, J. W. (2005). Social skills training for youth with emotional and behavioral disorders and learning disabilities: Problems, conclusions, and suggestions. Exceptionality, 13, 155-172.

Maag, J. W. (2006). Social skills training for students with emotional and behavioral disorders: A review of reviews. Behavioral Disorders, 32, 5-17.

Miller, M. J., Lane, K. L., \& Wehby, J. (2005). Social skills instruction for students with highincidence disabilities: A school-based intervention to address acquisition deficits. Preventing School Failure, 49(2), 27-39.

Moreno, G., Wong-Lo, M., Short, M., \& Bullock, L. M. (2014). Implementing a culturally attuned functional behavioural assessment to understand and address challenging behaviours demonstrated by students from diverse backgrounds. Emotional and Behavioural Difficulties, 19(4), 343-355.

Pierce, K., \& Schreibman, L. (1997). Multiple peer use of pivotal response training to increase social behaviors of classmates with autism: Results from trained and untrained peers. Journal of Applied Behavior Analysis, 30, 157-160.

Prater, M. A., Serna, L., \& Nakamura, K. K. (1999). Impact of peer teaching on the acquisition of social skills by adolescents with learning disabilities. Education and Treatment of Children, 22(1), 19-35.

Skiba, R. J., Simmons, A. B., Ritter, S., Gibb, A. C., Rausch, M. K., Cuadrado, J., \& Chung, C-G. (2008). Achieving equity in special education. Exceptional Children, 74, 264-288.

Spence, S. H. (2003). Social skills training with children and young people: Theory, evidence, and practice. Child and Adolescent Mental Health, 8, 84-96.

Strain, P. (2001). Empirically based social skill intervention: A case for quality-of-life improvement. Behavioral Disorders, 27(1), 30-36.

Terpstra, J., Higgins, K., \& Pierce, T. (2002). Can I play? Classroom-based interventions for teaching play skills to children with autism, Focus on Autism and Other Developmental Disabilities, $17,3-14$.

Trainor, A. A., \& Bal, A. (2014). Development and preliminary analysis of a rubric for culturally responsive research. The Journal of Special Education, 47(4), 203-216.

Waitoller, F., Artiles, A. J., \& Cheney, D. (2010). The miner's canary: A review of overrepresentation research and explanations. Journal of Special Education, 44, 29-49.

Wolf, M. M. (1978). Social validity: The case for subjective measurement, or how applied behavior analysis is finding its heart. Journal of Applied Behavior Analysis, 11, 203-214.

\section{Authors' Note}

Correspondence concerning this article should be addressed to Sunyoung Kim, University of Alabama, Box 870232, Tuscaloosa, AL, 35406, USA. Email: skim114@ua.edu 